\title{
4-Phenylbutyric Acid Reduces Endoplasmic Reticulum Stress, Trypsin Activation, and Acinar Cell Apoptosis While Increasing Secretion in Rat Pancreatic Acini
}

\author{
Antje Malo, PhD, * Burkhard Krüger, PhD,† Burkhard Göke, MD, * and Constanze H. Kubisch, MD*
}

\begin{abstract}
Objectives: Endoplasmic reticulum (ER) stress leads to misfolded proteins inside the ER and initiates unfolded protein response (UPR). Unfolded protein response components are involved in pancreatic function and activated during pancreatitis. However, the exact role of ER stress in the exocrine pancreas is unclear. The present study examined the effects of 4-phenylbutyric acid (4-PBA), an ER chaperone, on acini and UPR components.
\end{abstract}

Methods: Rat acini were stimulated with cholecystokinin $(10 \mathrm{pmol} / \mathrm{L}$ to $10 \mathrm{nmol} / \mathrm{L}$ ) with or without preincubation of 4-PBA. The UPR components were analyzed, including chaperone-binding protein, protein kinaselike ER kinase, $\mathrm{X}$-box-binding protein 1, c-Jun $\mathrm{NH}_{2}$-terminal kinase, CCAAT/enhancer-binding protein homologous protein, caspase 3 , and apoptosis. Effects of 4-PBA were measured on secretion, calcium, and trypsin activation.

Results: 4-Phenylbutyric acid led to an increase of secretion, whereas trypsin activation with supraphysiological cholecystokinin was significantly reduced. 4-Phenylbutyric acid prevented chaperone-binding protein up-regulation, diminished protein kinaselike ER kinase, and c-Jun $\mathrm{NH}_{2}$-terminal kinase phosphorylation, prohibited $\mathrm{X}$-box-binding protein 1 splicing and CCAAT/enhancer-binding protein homologous protein expression, caspase 3 activation, and apoptosis caused by supraphysiological cholecystokinin.

Conclusion: By incubation with 4-PBA, beneficial in urea cycle deficiency, it was possible to enhance enzyme secretion to suppress trypsin activation, UPR activation, and proapoptotic pathways. The data hint new perspectives for the use of chemical chaperones in pancreatic diseases.

Key Words: exocrine pancreatic acini, ER stress, 4-phenylbutyric acid, chemical chaperone

(Pancreas 2013;42: 92-101)

$T^{\text {he }}$ he exocrine pancreas is highly specialized in the production, storage, and release of inactive digestive enzymes (zymogenes), as well as a bicarbonate-rich fluid. To match the demand for digestive proteins, pancreatic acinar cells physiologically have the highest rate of protein synthesis among all adult human tissues. ${ }^{1}$ To adequately function, the pancreatic digestive proteins must fold into a distinct 3-dimensional arrangement and have to remain folded. Thus, the process of protein folding and maturation is a crucial step in the transmission of genetic

From the *Department of Medicine II, Campus Großhadern, University of Munich, Munich, Germany; and $\dagger$ Institute of Medical Biology, University of Rostock, Rostock, Germany.

Received for publication August 14, 2011; accepted March 5, 2012

Reprints: Constanze H. Kubisch, MD, Department of Internal Medicine,

Campus Großhadern, Marchioninistrasse 15, 81377 Munich, Germany (e-mail: Constanze.Kubisch@med.uni-muenchen.de).

This study was supported by the DGF grant KU 261/1-1 (to C.H. Kubisch).

The authors declare no conflict of interest.

Copyright (C) 2012 by Lippincott Williams \& Wilkins information into a specific biological function. Folding and stabilization take place inside the endoplasmic reticulum (ER) lumen after synthesis on membrane-bound ribosomes for a wealth of secretory proteins. After a protein enters the ER, it begins its chaperone-assisted folding and stabilization by multiple posttranslational modifications. ${ }^{2}$ To support its prominent role in the synthesis of digestive enzymes, the exocrine pancreatic acini have particularly abundant ER..$^{1,3}$

The chaperone-protein association provides an optimal protein-folding environment. It avoids uncontrolled and potentially harmful protein aggregation in the ER lumen. ${ }^{4}$ After correct protein folding, chaperones will dissociate and the protein in its locked tertiary structure exits the ER to move further along the secretory pathway. If the folding is incomplete or incorrect, export is inhibited by an ER quality control system. The proteins are retained in the ER, bound to a chaperone until the folding process is complete. An overly prolonged chaperon-protein association directs misfolded proteins to the proteosomal ERassociated protein degradation. ${ }^{5}$ The capacity of the ER to fold proteins is presumably limited by chaperone resources and can be exceeded by a high cellular protein demand during growth and differentiation, protein overexpression, and supraphysiological stimulation. These disturbances lead to the accumulation of partially folded or misfolded proteins in the ER, provoking ER stress response. ${ }^{6}$

Unfolded protein response (UPR) is the best-studied part of the ER stress response. It balances the folding capacity and folding demand within the organelle. It is achieved through an up-regulation of ER-resident chaperones, ER enlargement, down-regulation of gene transcription, and increase in ER-associated protein degradation. ${ }^{6-8}$ The UPR contains at least 3 distinct ER stress-sensing components located in the ER membrane appearing with a cytosolic and ER-luminal domain. It is activated in response to ER stress: the doublestranded RNA-activated protein kinaselike ER kinase (PERK), the activation transcription factor (ATF) 6, and the inositolrequiring protein (IRE) 1 . These molecules and stress sensors are associated with the luminal chaperone heavy chain-binding protein $(\mathrm{BiP})$, also known as glucose-related peptide 78 (GRP78) from the heat-shock family 70 . When proteins accumulate inside the ER, BiP preferentially associates with the unfolded proteins to assist folding. This results in the sequestration from and activation of the molecules and their downstream signaling partners. $^{9}$

Active PERK autophosphorylates, inhibits translation initiation, and prevents further influx of nascent proteins into an already saturated ER. ${ }^{6,10}$ Dissociation of BiP from IRE1 activates its ribonuclease activity. Once activated, the IRE1 cleaves the exonintron junctions of the basic leucine zipper domain transcription factor X-box binding protein (XBP) 1 mRNA. After ligation, the spliced XBP-1 (sXBP-1) encodes a transcriptionally active sXBP-1 protein. The sXBP-1 increases the size of the ER and elevates the levels of ER chaperones and folding enzymes. ${ }^{11,12}$ 
However, if these complex adaptive mechanisms are not sufficient and cells are exposed to prolonged and unbearable ER stress, the stress-damaged cells are eliminated through induction of apoptosis. Reports have shown that several moleculesincluding CCAAT/enhancer-binding protein homologous protein (CHOP), caspases, and molecules in the MAP kinase cascades - play a role in ER stress-induced apoptosis. ${ }^{13}$ CCAAT/ enhancer-binding protein homologous protein (also known as GADD153, a member of the CAAT/enhancer-binding protein family of basic leucine zipper domain transcription factors) transcription is induced by PERK in response to ER stress. CCAAT/ enhancer-binding protein homologous protein regulates the expression of $\mathrm{Bcl}-2$ family members, induces intracellular reactive oxygen species, and promotes cell-cycle arrest. ${ }^{14,15}$ Caspase 12 leads to apoptosis selectively in response to ER stress via activation of caspase $3 .{ }^{16}$ Caspase 3 can also be activated by BCL2associated agonist of cell death and BCL2-associated X protein activated by ER stress, and by up-regulation of NADH dehydrogenase and NADH oxidase induced by ER stress. ${ }^{17}$ Active IRE1 binds and clusters TNF receptor-associating factor 2 and apoptosis signal-regulating kinase 1 . Together, they phosphorylate c-Jun $\mathrm{NH}_{2}-$ terminal kinase (JNK). c-Jun $\mathrm{NH}_{2}$-terminal kinase, known to be phosphorylated in pancreatitis, activates downstream transcription factors, such as c-jun, c-fos, Sap-1, or regulates RNA stability. ${ }^{17-19}$

Acute pancreatitis (AP) ${ }^{20}$ arises initially in acinar cells but by incompletely understood mechanisms. ${ }^{21}$ Along with a plateau-shaped increase of intracellular calcium, starting at the apical zymogen-containing cell pole, a possible colocalization between lysosomal hydrolases and zymogenes may lead to a premature intracellular activation of trypsin and a blockage of secretion of the digestive enzymes. ${ }^{22,23}$ It occurs with local inflammation, apoptosis, necrosis, a loss of organ function and, in severe cases, with the development of a systemic inflammatory response syndrome. ${ }^{24}$

Solid evidence suggests that ER stress responses are involved in the early stages of AP. This includes dilatation or vacuolation of the ER and loss of membrane-bound ribosomes as one of the earliest morphological changes in AP. ${ }^{25-28}$ Endoplasmic reticulum changes seem to be a common reaction of acini to the induction of stress. Key regulators of the ER stress response are altered during AP, and several ER-resident chaperones are up-regulated. ${ }^{29,30}$

This study was aimed to determine if fatty acid with ER chaperone properties, 4-phenylbutyric acid (4-PBA), can reduce the ER stress with its consequences in rat pancreatic acinar cells. With the use of its ER stress-reducing potency, 4-PBA has successfully been used in the treatment of urea cycle deficiency and cystic fibrosis. ${ }^{31-33}$ To identify and characterize possible intracellular mechanisms, we investigated the results for 4-PBA pretreatment on calcium signaling, amylase secretion, and trypsin activation under conditions of physiological and pathophysiological cholecystokinin (CCK) stimulation in isolated rat acini.

\section{MATERIALS AND METHODS}

\section{Materials}

Essential amino acids were from GIBCO, Invitrogen (Carlsbad, Calif), chromatographically purified collagenase from Worthington Biochemical Corp (Lakewood, NJ), CCK from Research Plus (Manasquan, NJ), 4-PBA from Calbiochem (Darmstadt, Germany).

\section{Acinar Amylase Secretion}

Phadebas amylase test was purchased from Magle Life Sciences International (Sweden).

\section{Acinar Trypsin Activity}

Protein assay was from Bio-Rad Laboratory Inc (Hercules, Calif), Substrat Boc-Gln-Ala-AMC $\cdot \mathrm{HCl}$ from Bachem (Bubendorf, Switzerland), purified trypsin for standards from Sigma-Aldrich (St Louis, Mo).

\section{Western Blotting}

Molecular weight marker and protein assay and blotting cells were purchased from Bio-Rad Laboratory Inc; proteinase inhibitor from Merck Calbiochem (Darmstadt, Germany); nitrocellulose membrane Protean from Whatman (Maistone, UK); antiphospho-PERK antibody (sc-32577-R), anti-XBP-1 antibody (sc-7160), and anti-Caspase 3 antibody (sc-7148) from Santa Cruz Biotechnology (Santa Cruz, Calif); anti-BiP antibody (\#SPA-826) from Stressgen (Ann Arbor, MI); anti-CHOP (ab11419) from Abcam (Cambridge, UK), antiphospho JNK (\#9251) from Cell Signaling (Danvers, Mass); anti-Actin anti body (A5441) from Sigma-Aldrich; secondary horseradish peroxidase-conjugated antibodies from GE Healthcare (Bucks, UK).

\section{Intracellular Calcium Measurement}

Fura-2-acetoxuymethyl ester was from Molecular Probes (Eugene, Ore), and thapsigargin (T9033) was from SigmaAldrich).

\section{Annexin V Staining}

The ApoAlert annexin V-FITC apoptosis kit (\#630109) was purchased from Clontech (Mountain View, Calif).

\section{Methods}

\section{Preparation of Isolated Acini}

The preparation of pancreatic acini was carried out as previously described. ${ }^{34}$ Briefly, pancreata from male Wistar rats (150-200 g; Charles River Laboratories Inc, Sulzfeld, Germany) were removed and immediately digested by collagenase at $37^{\circ} \mathrm{C}$ under an atmosphere of $95 \% \mathrm{O}_{2}$ and $5 \% \mathrm{CO}_{2}$, mechanically dispersed, and passed through a $150-\mu \mathrm{m}$ mesh nylon cloth. Acini were purified by an albumin gradient containing $4 \%$ bovine serum albumin and resuspended in an Erlenmeyer flask in hydroxyethyl piperazineethanesulfonic acid-Ringer buffer (supplemented with $0.1 \%$ bovine serum albumin, $0.01 \%$ trypsin inhibitor $\mathrm{pH}$ 7.4) under the atmosphere of $100 \% \mathrm{O}_{2}$.

In control experiments, basal levels of stress kinases and other phosphorylation-dependent pathways are activated to a limited extent in isolated, dispersed acini. Therefore, the isolated acini were preincubated for 2 hours in a shaking water bath at $37^{\circ} \mathrm{C}$ before the experiments. 4-Phenylbutyric acid was dissolved in aqua bidest. The isolated cells were split and preincubated for 2 hours with or without 3.75-nmol/L 4-PBA. Dispersed acini were stimulated with different concentrations of CCK (10 pmol/L to $10 \mathrm{nmol} / \mathrm{L}$ ) for 30 minutes in $2-\mathrm{mL}$ aliquots in duplicates for each experiment. All experiments were carried out at $37^{\circ} \mathrm{C}$ and repeated at least 4 times, with acini isolated from different animals.

\section{Quantification of Pancreatic Acini Amylase Secretion}

After CCK stimulation, acini were spun down and the supernatant was used to quantify amylase release as previously described. ${ }^{35}$ Net stimulated secretion of amylase in percent was calculated by subtracting the secretion in the absence of secretagogue ("basal" secretion) from the secretion of amylase noted in the presence of CCK ("stimulated" secretion). Additionally, 
the total amount of amylase in acinar cells was determined to express the amount of amylase secretion in percent of total amylase.

\section{Measurement of Intracellular Calcium}

Intracellular calcium concentrations were determined using the calcium-sensitive fluorescent dye fura- 2 acetoxymethylester. Acini were loaded with $1-\mu \mathrm{mol} / \mathrm{L}$ fura- 2 acetoxymethylester for 25 minutes at room temperature. After washing, the cells were incubated with 3.75-n mol/L 4-PBA. To further investigate the mechanism of action for 4-PBA, sets of acinar cells were incubated in $10-\mu \mathrm{mol} / \mathrm{L}$ thapsigargin. Measurements were performed with a radio imaging system (TILL Photomics, Gräfelfing, Germany) using excitation wavelengths of 340 and $380 \mathrm{~nm}$, and emitted light was collected at $510 \mathrm{~nm}$ according to Mooren et al. ${ }^{36}$

\section{Trypsin Activity in Pancreatic Acini}

After stimulation, the cell pellet was resuspended in icecold MOPS buffer and homogenized using a Teflon and glass homogenizer. The homogenate was used to determine the trypsin activity as described. ${ }^{35}$ The concentration was calculated using standards generated by purified trypsin. Protein concentrations of each sample were determined, and trypsin activity was expressed as femtomol per milligram $(\mathrm{fmol} / \mathrm{mg}$ ) protein.

\section{Western Blotting of Pancreatic Acini Proteins}

Whole cell lysates were prepared from acini and used for Western blotting as described previously. ${ }^{35}$ Antibodies were used in the following concentrations: anti-BiP, 1:1000; antiphosphoPERK, 1:250; anti-XBP-1, 1:500; anticaspase 3, 1:500; antiCHOP, 1:500; antiphospho-JNK, 1:2,000; and anti-Actin, $1: 5,000$, as internal loading control. Membranes were incubated with the appropriate $\operatorname{IgG}$ horseradish peroxidase-conjugated secondary antibody $(1: 10,000)$. Antibody binding was detected by chemiluminescence radiography. Membranes were scanned, recorded digitally, and processed using ImageJ software.

\section{Early Stages of Apoptosis and Necrosis in Pancreatic Acini}

The extent of early apoptosis and necrosis in acinar cells was determined by annexin V- fluorescein isothiocyanate/propidium iodide (PI) staining according to the manufacturer's instruction, using $5-\mu \mathrm{L}$ annexin V and $10-\mu \mathrm{L}$ PI.

\section{Statistical Analysis}

Cholecystokinin stimulation was performed in duplicates, and all experiments were repeated at least 4 times using a different acini preparation each time. The Student $t$ test was used to establish the difference of each parameter. Results were regarded as significantly different when $P<0.05$. Values are expressed as mean \pm SE.

The local Animal Care and Use Committee (University of Munich) approved all animal experimental protocols. Rats were treated according to the Guiding Principles in the Care and Use of Animals.

\section{RESULTS}

\section{Dose Finding of 4-PBA}

4-Phenylbutyric acid has been used in different cells, such as adipocytes and bronchial and intestinal endothelial cells with concentrations from 0.05 to $20 \mathrm{mmol} / \mathrm{L} .{ }^{37-39}$ So far, 4-PBA was not used in exocrine pancreatic acini. Therefore, we tested 4PBA for preincubation in 3 different concentrations $(2.5,5$, and $10 \mathrm{nmol} / \mathrm{L}$ ) and stimulated the acini thereafter with different CCK concentrations ( $100 \mathrm{pmol} / \mathrm{L}$ or $10 \mathrm{nmol} / \mathrm{L})$. Subsequently, amylase secretion was measured in all groups and compared to controls (acini only stimulated with CCK; Fig. 1). We further measured $\mathrm{BiP}$ expression and PERK phosphorylation in Western blotting from whole cell lysates after CCK stimulation (data not shown). Five-nanomole per liter 4-PBA was the most effective dose on secretion, BiP, and PERK regulation. However, because it is known from the literature that 4-PBA may have a possible inhibitory effect on secretion starting at $5 \mathrm{nmol} / \mathrm{L}$ and higher, ${ }^{40}$ we choose 3.75-nmol/L 4-PBA for all subsequent experiments. Incubation of acinar cells with 4-PBA alone without CCK stimulation caused neither amylase secretion nor trypsin activation (Fig. 1, extreme left bars).

\section{Effects of 4-PBA Preincubation on Secretion and the Intracellular Calcium Concentration}

First, we evaluated the effects of 4-PBA on amylase secretion in isolated pancreatic acini after 30 minutes of incubation with various concentrations of CCK (Fig. 2A). Cholecystokinin without 4-PBA preincubation caused a dose-dependent stimulation of amylase secretion at lower concentrations and subsequent inhibition at higher concentrations, according to previously reported observations with $\mathrm{CCK}^{41}$ In contrast, preincubation with 4-PBA led to an increased amylase secretion starting

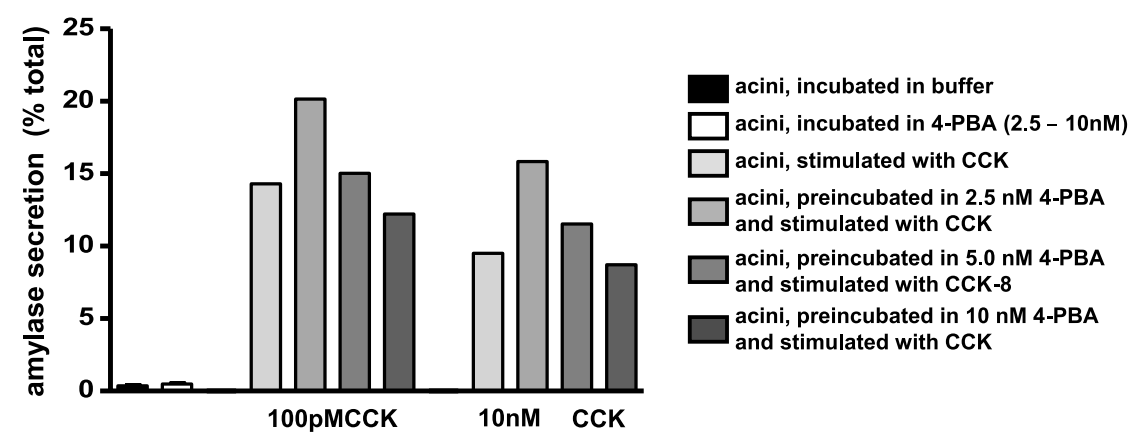

FIGURE 1. Dose finding for 4-PBA. Pancreatic acinar cells were first incubated with the three 4-PBA concentrations or not (black and white bar on the very left). Thereafter, pancreatic acini were preincubated with $2.5-, 5.0-$, or $10-\mathrm{nmol} / \mathrm{L} 4-\mathrm{PBA}$ at $37^{\circ} \mathrm{C}$ and thereafter stimulated with either 100 -pmol/L CCK or 10-nmol/L CCK over 30 minutes. Control acini were just stimulated with CCK without 4-PBA preincubation. Amylase secretion was measured in the cell supernatant and expressed in percent of total amylase. Each bar in the figure represents the mean of 3 independent experiments. 

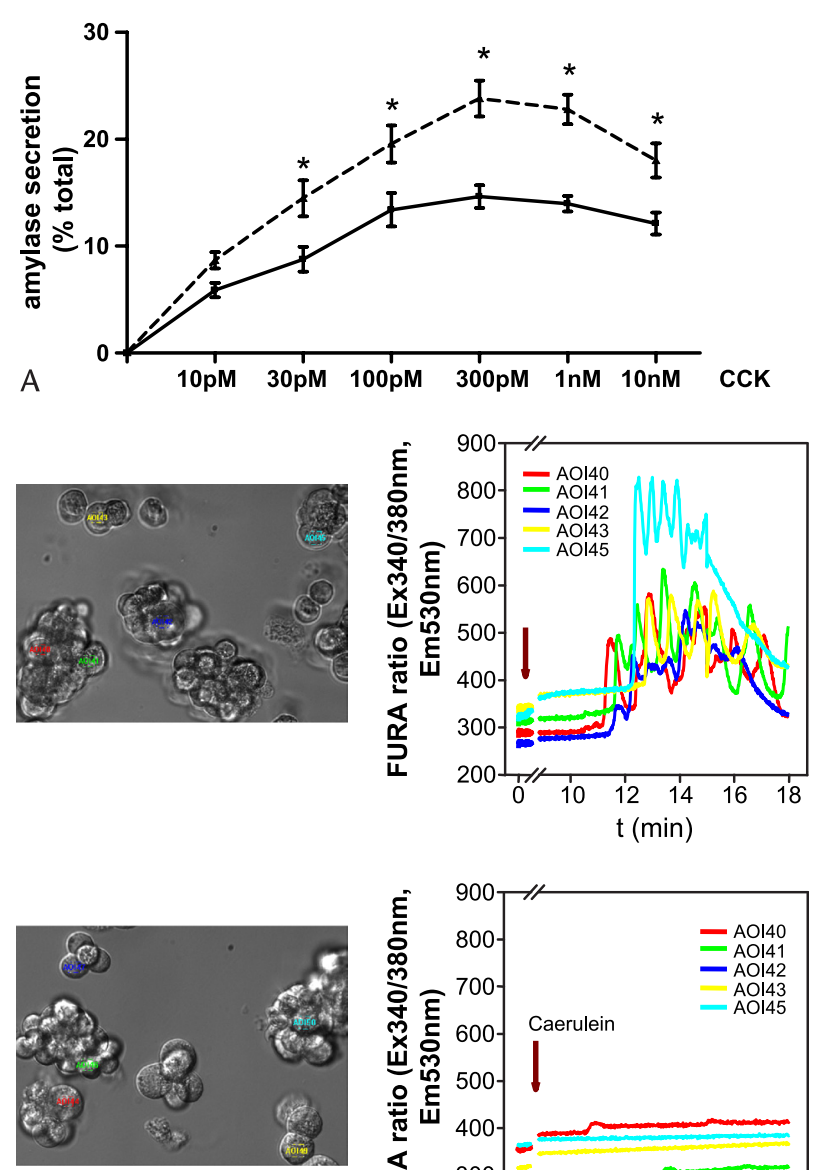

B

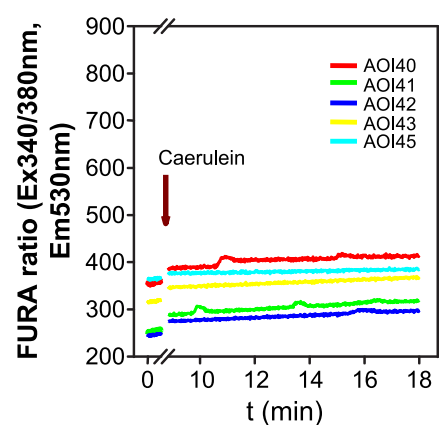

FIGURE 2. Effects of 4-PBA preincubation on amylase secretion and cytoplasmic calcium concentration. A, Acinar amylase secretion after 30 minutes of CCK incubation. CCK caused a dose-dependent amylase secretion at lower and inhibition at higher concentrations and maximal effects at 300-pmol/L CCK. Starting at a $30 \mathrm{pM} \mathrm{CCK}$ the 4-PBA preincubation (dashed line) let to a significant higher amylase secretion compared to the cells just stimulated with CCK. Further, the secretory inhibition caused by supraphysiological CCK is less pronounced after 4-PBA treatment. Each point in the graphs represents the mean + SE of 8 independent experiments for amylase secretion, each performed in duplicate. Asterisks indicate the $P<0.05$ when compared to CCK stimulation alone. B, Effect of 4-PBA on cytoplasmic calcium. Fura-2 loaded acini were incubated with (lower panel) or without (upper panel) $3.75 \mathrm{nM} 4-\mathrm{PBA}$ at room temperature and stimulated with $10 \mathrm{nM}$ CCK. Measurements were performed with a TILL Photomics Polychrome II radio imaging system from different areas of interest (AOI, right panel). The left panel shows the histology of the analyzed acini. The quantification of 5 different measurements over 5 minutes are demonstrated on the right panel.

at 30-pmol/L CCK with maximal effects at a 300-pmol/L CCK stimulation (maximal, $23.82 \%$ of total amylase \pm 1.68 vs $13.42 \%$ of total amylase \pm 1.49 without preincubation). Further, the secretory inhibition caused by supraphysiological concentrations of CCK $(>1 \mathrm{nmol} / \mathrm{L})$ was apparent but less pronounced after 4-PBA treatment. Basal secretion of acinar cells incubated with 4-PBA did not differ between the groups.
An oscillating increase in intracellular calcium is the primary driver of enzyme secretion in pancreatic acinar cells, for example, amylase. ${ }^{42}$ It is known that low-dose CCK causes spikes of calcium release from intracellular calcium stored at the apical cell pole and coincidently stimulates secretion. High CCK concentrations ( $1 \mathrm{nmol} / \mathrm{L}$ and higher) induce an increase in intracellular calcium followed by a sustained plateau-shaped elevation of cytoplasmic calcium. This long-lasting calcium signal without return to baseline inhibits cellular functions, such as secretion, and leads to intracellular trypsinogen activation. In our study, it was tested whether 4-PBA preincubation changes the calcium signal in acinar cells and if this may be responsible for an increase in amylase secretion. Therefore, isolated pancreatic acini were loaded with the calcium-sensitive fluorescent dye fura-2 in the presence of 4-PBA. 4-Phenylbutyric acid incubation alone over a period of two hours did not change the intracellular calcium level (data not shown). Acinar cell stimulation with 10$\mathrm{nmol} / \mathrm{L}$ CCK over 30 min caused a large intracellular calcium mobilization in acinar cells after CCK administration (Fig. 2B, upper panel). Hereby, the cytoplasmic calcium increase was first noted at the basal cell pole. Preincubation with 4-PBA and the sequential stimulation of acinar cells with 10-nmol/L CCK showed a very small and continuous intracellular calcium mobilization in acinar cells over 18 minutes. The increase over time is very little and mostly due to membrane leakage caused by the preparation procedures. 4-Phenylbutyric acid blocks the pathophysiological plateau-shaped calcium signal and modifies it (Fig. 2B, lower panel). To further investigate the way 4-PBA acts on calcium signaling acinar cells, we incubated with $10-\mu \mathrm{mol} / \mathrm{L}$ thapsigargin, an agent that specifically inhibits the sarcoendoplasmic reticulum calcium ATPase (SERCA) leading to an IP3independent intracellular calcium increase. ${ }^{43}$ After adding thapsigargin to untreated acinar cells, an immediate and huge increase in intracellular calcium was measured (Fig. 3A). 4Phenylbutyric acid pretreated cells do not show any increase in intracellular calcium after adding the SERCA inhibitor (Fig. 3B).

\section{Effects of 4-PBA Preincubation on Intracellular Trypsin Activation}

Furthermore, the effect of 4-PBA was tested on trypsin activation, the digestive enzyme in acinar cells that can activate all other zymogens and contribute to acinar cell damage. Preincubation with 4-PBA had significant effects on the activation of intracellular trypsin in rat acini after CCK stimulation (Fig. 4A). CCK alone increased trypsin activity in a concentration-dependent manner. An increase of trypsin activity compared to unstimulated cells was noted at 100-pmol/ L CCK. Trypsin activity was further increased by higher concentrations to a maximum of $159.95 \pm 14.86-\mathrm{fmol} / \mathrm{mg}$ protein (equals a $414 \%$ increase compared to unstimulated acini) at $10-\mathrm{nmol} / \mathrm{L}$ CCK stimulation. Pretreatment with 4PBA caused a significant reduction of trypsin activation up to $92.81 \pm 8.06-\mathrm{fmol} / \mathrm{mg}$ protein at supraphysiological CCK stimulation.

\section{Effects of Tauroursodeoxycholic Acid Preincubation on Components of the UPR}

As a member of the heat-shock protein family 70 , BiP is an abundant ER-specific chaperone, and its level is very sensitive to ER stress. Therefore, we investigated the effects of 4-PBA on $\mathrm{CCK}$-induced $\mathrm{BiP}$ expression in acini by Western blotting. The effects of $\mathrm{CCK}$ on $\mathrm{BiP}$ expression were dose dependent, with apparent effects at 100-pmol/L CCK, and a maximum increase up to $376.67 \% \pm 49.46$ compared to unstimulated cells at 

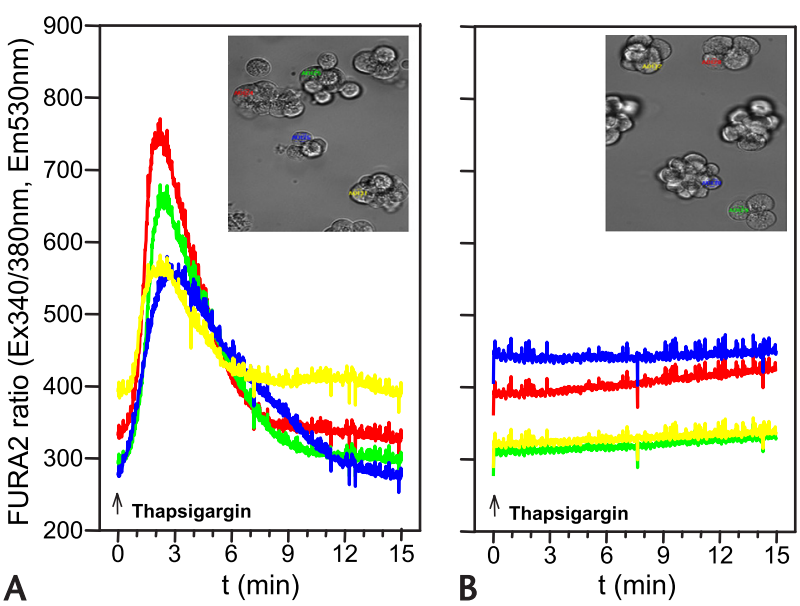

FIGURE 3. Effects of thapsigargin and 4-PBA preincubation on cytoplasmic calcium concentration. A, Intracellular calcium signal of 4 isolated, untreated acinar cells that were incubated with $10-\mu \mathrm{mol} / \mathrm{L}$ thapsigargin. B, Intracellular calcium signal of another set of 4 isolated and 4-PBA-pretreated acinar cells that also were incubated with the same concentration of thapsigargin. Measurements were performed with a TILL Photomics Polychrome II radio imaging system from different areas of interest (AOI). In the right corner of each graph, a histological picture of the analyzed acinar cells is presented.

1-nmol/L CCK and a little decline thereafter (Figs. 4B, C). Acini preincubated with 4-PBA showed no significant increase of BiP expression at any $\mathrm{CCK}$ concentration. Expression of $\mathrm{BiP}$ was diminished by 4-PBA in response to physiological and supraphysiological CCK stimulations and therefore showed a significant reduction at $300-\mathrm{pmol} / \mathrm{L}$ up to $10-\mathrm{nmol} / \mathrm{L} \mathrm{CCK}$ stimulation.

Protein kinaselike ER kinase is one of the major sensors and transducers of the ER stress, localized to the ER membrane and kept inactive by the ER luminal binding to BiP. When ER stress occurs, BiP binding shifts to unfolded proteins and leads to its dissociation from PERK with subsequent PERK autophosphorylation. ${ }^{44}$ Therefore, we used a phospho-specific PERK antibody to determine its activation status after CCK treatment (Fig. 5B). CCK alone caused an increase in acinar PERK phosphorylation, with effects starting at 30-pmol/L CCK. The maximum increase in PERK phosphorylation was observed after a stimulation with $100-\mathrm{pmol} / \mathrm{L}$ CCK $(816.38 \% \pm 150.54 \%$ of unstimulated control cells). 4-Phenylbutyric acid pretreated cells showed only a small but not significant increase in phosphoPERK after 100-pmol/L CCK stimulation compared to controls. Protein kinaselike ER kinase phosphorylation was significantly reduced in the 4-PBA-treated cells after CCK stimulation starting at $100-\mathrm{pmol} / \mathrm{L}$ up to $1-\mathrm{nmol} / \mathrm{L}$ CCK.

Inositol-requiring protein 1 is a second ER stress sensor and transducer, bound to BiP under physiological conditions. Upon stress, IRE1 becomes active as an endonuclease. Active IRE1 targets cytoplasmic XBP-1 mRNA and generates a splice variant that converts XBP-1 into SXBP-1, an active transcription factor. Subsequently, sXBP-1 induces the transcription of several ER stress-related genes involved in the biogenesis of the organelle itself. For our experiments, we analyzed the $28-\mathrm{kd}$ small splice variant sXBP-1 to describe changes in IRE1 activity (Figs. 6A, B). CCK stimulation caused a significant increase in XBP-1 splicing compared to unstimulated acini. Spliced XBP-1 expression increased with 30-pmol/L CCK-8 and peaked at $300 \mathrm{pmol} / \mathrm{L}$, with an increase of $363.58 \% \pm 53.42 \%$ of unstimulated acini and a decline thereafter. Preincubation with 4-PBA led to a sig- nificant inhibition of XBP-1 splicing starting at $100 \mathrm{pmol} / \mathrm{L}$ up to $1 \mathrm{nmol} / \mathrm{L}$.

CCAAT/enhancer-binding protein homologous protein is a proapoptotic transcription factor that is produced during prolonged or severe ER stress. ${ }^{14}$ Hence, an increase of CHOP is associated with increased cell death. We evaluated the expression of CHOP by Western blotting after the stimulation by various concentrations of CCK. CCK-induced concentration-dependent CHOP expression (Figs. 7A, B). A significant effect was first observed at 30-pmol/L CCK compared to unstimulated acini, with its maximal effect of a $379.39 \% \pm 78.91 \%$ increase of unstimulated control cells. In contrast, 4-PBA preincubation inhibited completely the expression of $\mathrm{CHOP}$ at any concentration. The difference in $\mathrm{CHOP}$ expression between acinar cells and 4-PBA-pretreated acinar cells was significant at $30 \mathrm{pmol} / \mathrm{L}$ up to $1 \mathrm{nmol} / \mathrm{L}$.

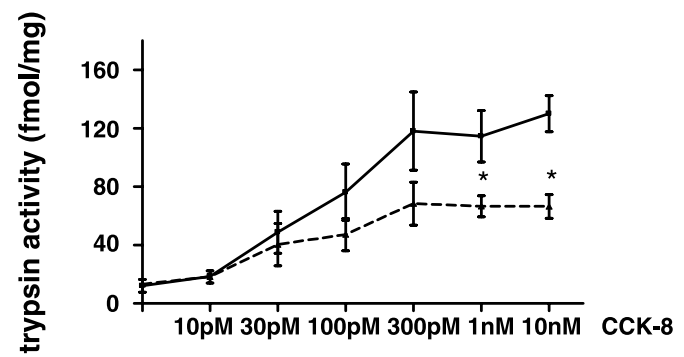

A
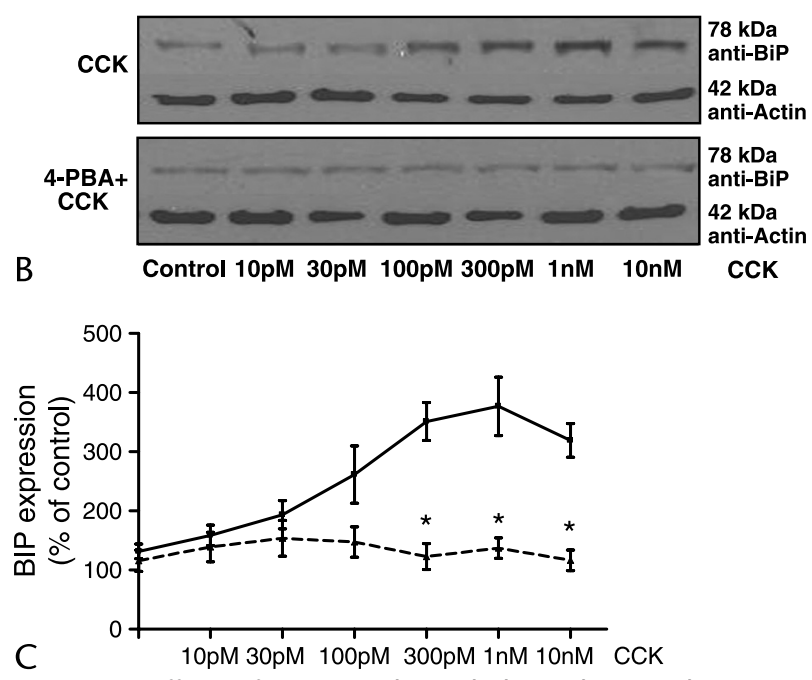

FIGURE 4. Effects of tauroursodeoxycholic acid preincubation on trypsinogen activation and BiP. A, Effect of 4-PBA preincubation on the intracellular trypsin activity in rat acini after CCK stimulation. Acinar cells were either stimulated by CCK alone (solid line) or pretreated for 30 minutes with 3.75-nmol/L 4-PBA (dashed line). Each point of the graphs represents mean \pm SE of 4 independent experiments. Asterisks indicate $P<0.05$ compared to CCK alone. Induction of BiP as ER stress-inducible chaperone was analyzed by Western blotting from acinar cell lysates with or without preincubation with $3.75-\mathrm{nmol} / \mathrm{L} 4-\mathrm{PBA}$ and CCK stimulation. B, Representative Western blots of 5 individual experiments. For quantification, Western blot membranes were counterstained with antiactin (lines 2 and 4). C, Quantitative Western blot data from 5 independent experiments. Results from CCK stimulation after 4-PBA preincubation are shown in the dashed line. Asterisks indicate $P<0.05$ compared to CCK stimulation alone. 


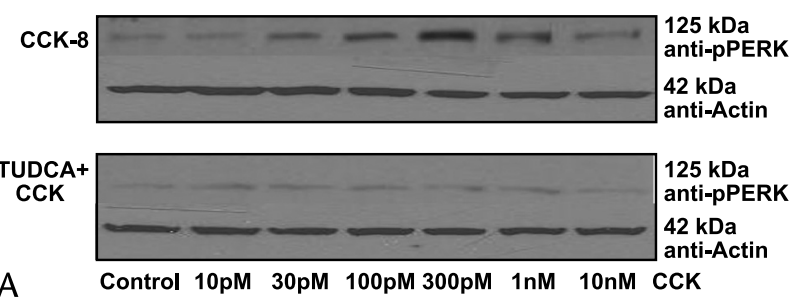

A Control 10pM 30pM 100pM 300pM 1nM 10nM CCK

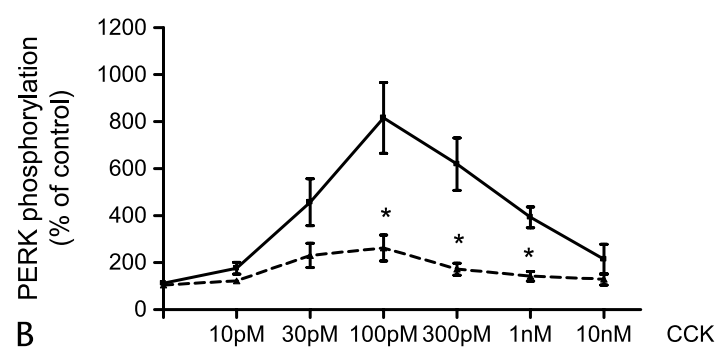

FIGURE 5. Effects of 4-PBA preincubation on PERK

phosphorylation. A, Representative Western blots stained against phospho-PERK of 5 individual experiments. Results after 4-PBA pretreatment are shown in a dashed line. Each point represents mean \pm SE. Asterisks indicate $P<0.05$ compared to CCK stimulation alone.

Another proapoptotic pathway emanating from the ER involves the ER initiator procaspase 12 , which is activated by cleavage under prolonged ER stress conditions. It can activate the effector caspase 3, leading to apoptosis. Using Western blotting from CCK-stimulated acinar cells, we investigated the expression of the 17-kd cleavage product of active caspase 3 (Figs. 8A, B). Our results show a dose-dependent increase in activated caspase 3 after CCK stimulation. It peaks at the 1-nmol/ L CCK with a maximal increase up to $617.57 \% \pm 107.14 \%$ compared to unstimulated acini and declines thereafter. With the 4-PBA preincubation, caspase 3 activation was not detected at any CCK concentration. 4-Phenylbutyric acid treatment prevented completely caspase 3 activation in rat acini.

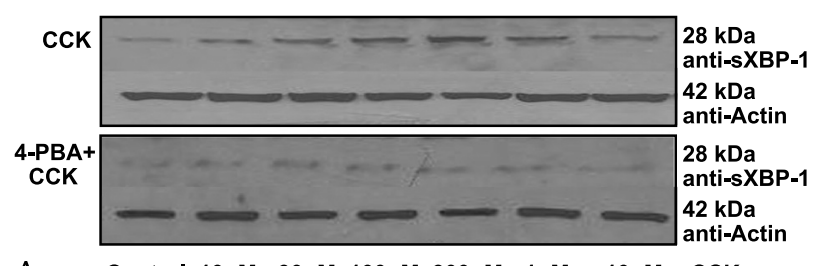

A

Control 10pM 30pM 100pM 300pM 1nM 10nM CCK

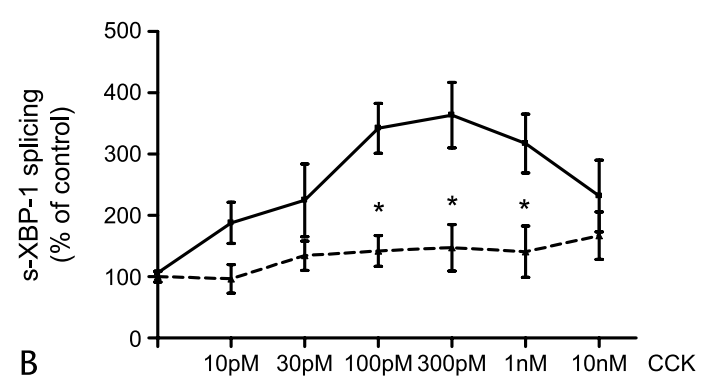

FIGURE 6. Effects of 4-PBA preincubation on XBP-1 splicing. A, Representative Western blots of 5 individual experiments. $B$, Quantitative analysis of the Western blot data from 5 independent experiments. Results after 4-PBA pretreatment are shown in a dashed line. Each point represents mean \pm SE. Asterisks indicate $P<0.05$ compared to CCK stimulation alone.

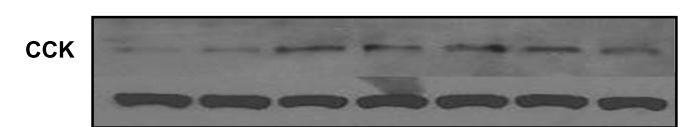

$31 \mathrm{kDa}$ anti-CHOP $42 \mathrm{kDa}$ anti-Actin

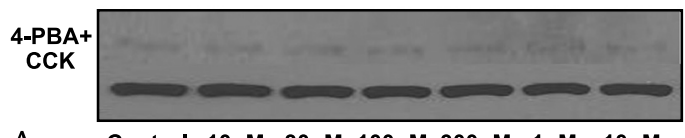

$31 \mathrm{kDa}$ anti-CHOP $42 \mathrm{kDa}$ anti-Actin

A

Control 10pM 30pM 100pM 300pM 1nM 10nM CCK

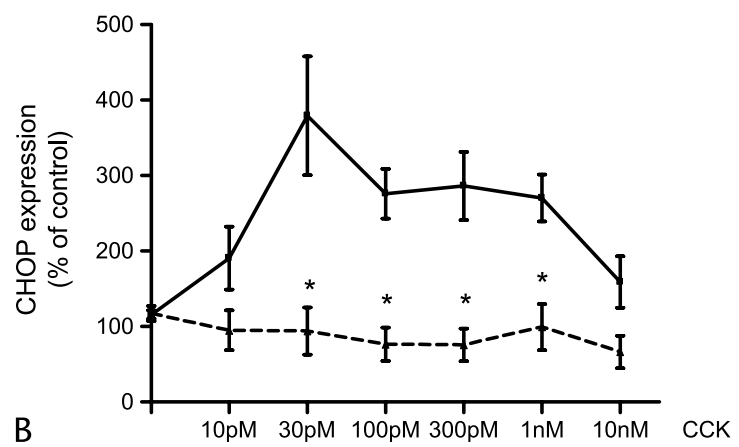

FIGURE 7. Effects of 4-PBA preincubation on ER stress-associated CHOP induction. A, Representative Western blots of 5 individual experiments. B, Quantitative Western blot data. Results after 30-minute pretreatment with $3.75-\mathrm{nmol} / \mathrm{L}$ 4-PBA are shown in the dashed line. Each point represents mean \pm SE of 5 individual experiments. Asterisks indicate $P<0.05$ compared to CCK stimulation alone.

We also analyzed the phosphorylation of JNK. Activation of IRE1 can lead via binding and clustering of TRAF2 to phosphorJNK. Several isoforms are known with different molecular weights: JNK 1 (46 kd) and JNK 2/3 (54 kd). Using Western blotting with a phospho-specific JNK antibody, we investigated the phosphorylation status of JNK in pancreatic acini after CCK stimulation with or without 4-PBA pretreatment (Fig. 9A). For
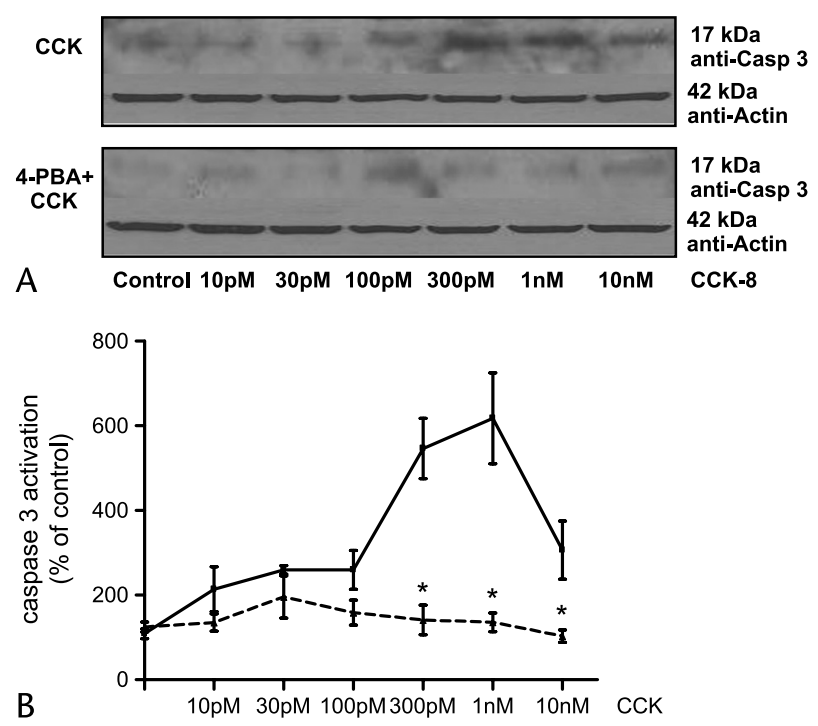

FIGURE 8. Effects of 4-PBA preincubation on ER stress associated caspase 3 activation. A, Representative Western blots of 4 individual experiments. B, Quantitative Western blot analysis from 4 individual experiments. Results after 4-PBA pretreatment are shown in the dashed line. Each point represents mean \pm SE of 4 independent experiments. Asterisks indicate $P<0.05$ compared to CCK stimulation alone. 


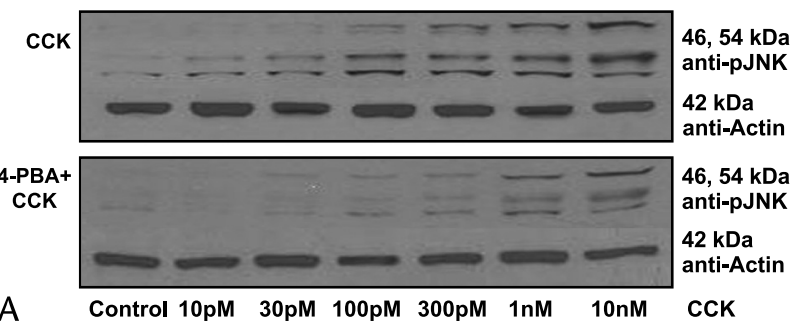

A

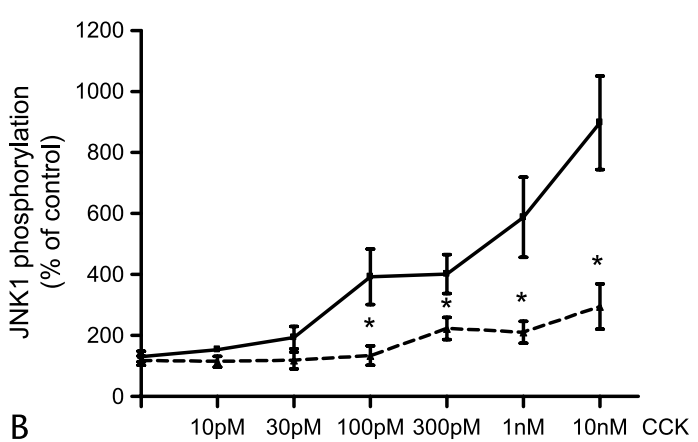

FIGURE 9. Effects of 4-PBA preincubation on ER stress associated JNK phosphorylation. A, Representative Western blot of an experiment repeated 4 times. B, Quantitative Western blot data from 4 individual experiments. Results after 4-PBA pretreatment are shown in the dashed line. Each point represents mean \pm SE. Asterisks indicate $P<0.05$ compared to CCK stimulation alone.

the quantification of our data, we analyzed the levels of phosphoJNK1 exemplary (Fig. 9B). As described earlier, ${ }^{35}$ JNK phosphorylation increases after CCK stimulation, with minimal effects observed at $30-\mathrm{pmol} / \mathrm{L} \mathrm{CCK}$. We observed maximal effects at the supraphysiological concentration of $10-\mathrm{nmol} / \mathrm{L}$ CCK (up to $898.02 \% \pm 153.641 \%$ of unstimulated cells). 4Phenylbutyric acid pretreatment was not able to abolish JNK phosphorylation but reduced it significantly $(294.91 \% \pm$ $74.56 \%)$.

To measure early stages of apoptosis in acinar cells, we determined the phosphatidylserine translocation to the outer cell membrane by annexin V staining. We used PI to determine necrosis in acinar cells. Acinar cell stimulation with CCK led to a dose-dependent increase in annexin $\mathrm{V}$ staining, with its maximum at 300 -pmol/L CCK $(81.3 \pm 9.1$ relative fluorescent units [RFU] per microgram of protein) and a decrease thereafter (Fig. 10A). If cells were preincubated with 4-PBA and then stimulated by CCK, significant less apoptotic signals were found (43.05 RFU per microgram of protein at 300-pmol/L CCK stimulation). This was mirrored when measuring necrosis in acinar cells using PI. Maximal necrosis was measured after stimulation with $300-\mathrm{pmol} / \mathrm{L}$ of CCK $(56.67 \pm 7.47 \mathrm{RFU}$ per microgram of protein) and was significantly reduced after 4PBA pretreatment at $100-\mathrm{pmol} / \mathrm{L}$ up to $1-\mathrm{nmol} / \mathrm{L}$ CCK (maximal effect at 300-pmol/L CCK stimulation: $36.72 \pm 5.29 \mathrm{RFU}$ per microgram of protein; Fig. 10B).

\section{DISCUSSION}

Endoplasmic reticulum stress and its responses are relevant in the development of acute pancreatitis. Histological examinations demonstrate early on morphological changes of the ER during the onset of AP. Gene-profiling studies show significant alterations in ER stress key regulators and in a model of necrotizing pancreatitis all ER stress sensors-PERK, IRE1, and
ATF6 - become activated, and the initiation of downstream signal pathways occurs initiated from the ER. ${ }^{25-30}$

Following the principle of ER function and stress, we assume that CCK in physiological concentrations stimulates ER function and increases protein folding, zymogen production, and secretion in acini. Supraphysiological stimulation leads to the exceedance of the ER function and folding capacity and with this to an unorganized accumulation of only partly finished zymogens inside the organelle. It prompts the activation of the ER stress sensors PERK, IRE1, and ATF6, resulting in a general stop of transcription and translation, induction of chaperones, expansion of the ER itself, and induction of protein degradation to clear the unfinished folded proteins, trying to match the demands of protein folding and secretion from the acinar cell upon stimulation as the physiological part of the UPR. As pathophysiological part of the UPR, several proapoptotic signals and autophagy can be activated, emitting from the ER for a programmed cell death to eliminate the deadly injured cell in an organized manner for the protection of the neighboring cells and the whole tissue. ${ }^{45,46}$

In vitro, several secretagogues were able to induce the ERspecific chaperone BiP and XBP-1 splicing in a concentrationdepending manner. CCK, in supraphysiological doses induces acinar cell damage, inhibits amylase secretion, activates trypsin, and induces proapoptotic ER signals. ${ }^{34}$ Here, we investigated ER function in response to CCK treatment on a fatty acid with chemical chaperone properties. We aimed to interfere with the pathophysiological toxic ER signaling introducing 4-PBA pretreatment as a powerful antistress principle.
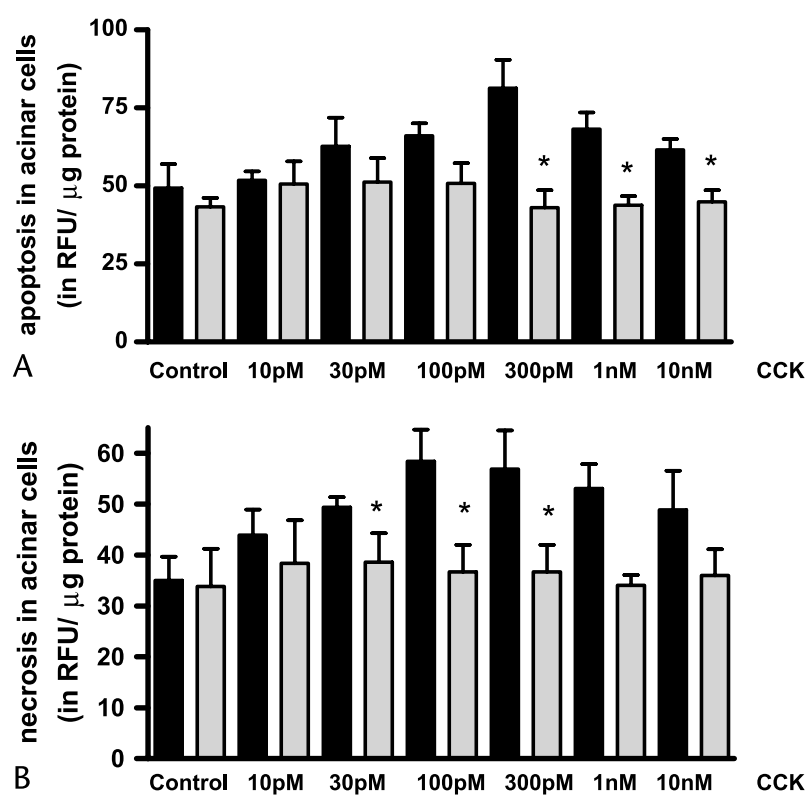

FIGURE 10. Effects of tauroursodeoxycholic acid preincubation on annexin V-fluorescein isothiocyanate and $\mathrm{PI}$ staining in pancreatic acinar cells. Isolated acini were quantified by fluorescent plate reader with dual filter set for fluorescein isothiocyanate (excitation, $488 \mathrm{nmol} / \mathrm{L}$ emission, $530 \mathrm{nmol} / \mathrm{L}$ ) and $\mathrm{PI}$ (excitation, $535 \mathrm{nmol} / \mathrm{L}$ emission, $617 \mathrm{nmol} / \mathrm{L}$ ) after 30-minute preincubation with 3.75-nmol/L 4-PBA followed by stimulation with different concentrations of CCK (gray bars, A and B) after stimulation by CCK alone (black bars, A and B). Data were expressed as RFU

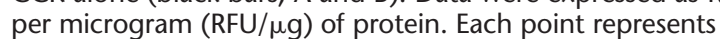
mean \pm SE of 4 independent experiments. Asterisks indicate $P<0.05$ compared to CCK stimulation alone. 
The knowledge about the ER chaperone comes from DNA point mutations that result in the production of misfolded proteins that are transcribed and translated at normal levels but, owing to the ER quality control system, are unable to reach their functional designation. Misfolded proteins are retained inside the ER and induce ER stress. Examples for misfolding diseases are Alzheimer disease and cystic fibrosis and $\beta$-thalassemia. ${ }^{42,47,48}$ Thus, although still exhibiting some biological activity with minor mutations, the mutants are retained in the ER. Folding intermediates form fibrillar aggregates, react to harmful substances, or can induce apoptosis of the stressed cell. The same can happen during phases of an increased protein demand, for example, during growth and differentiation or defense of bacterial or viral infections and overstimulation. ${ }^{49-51}$ Therefore, extraordinary efforts have been made to design therapeutic interventions that prevent or correct the structural abnormality of disease-causing misfolded proteins. In this regard, rescue of "trafficking defective" proteins or optimization of ER function in times of overstimulation by pharmacological or chemical chaperones including 4-PBA is emerging as one of the most promising therapeutic strategies for such disorders. ${ }^{52-54}$

In the recent years, ER stress has been implicated in metabolic disorders such as obesity, type 2 diabetes, and atherosclerosis. ${ }^{55}$ Animal studies show a reduction in ER stress and its consequences as well as the disease symptoms via the administration of chemical chaperones by mechanisms of restoring ER function. Chemical chaperones are small molecules that are nonselective in their ability to stabilize mutant proteins to facilitate their proper folding, slightly reminiscent of the chaperoning function of the many intracellular molecular chaperones. Chemical chaperones are usually osmolytically active such that they equilibrate cellular osmotic pressure. These osmolytes are compatible with protein function and can act as chemical chaperones by increasing the stability of native proteins and assisting refolding of unfolded or misfolded proteins and affecting protein trafficking. ${ }^{55}$

Özcan et $\mathrm{al}^{56,57}$ describe 4-PBA as a short-chain fatty acid with chaperone properties. In hepatoma cells, 4-PBA-suppressed ER stress-induced phosphorylation of PERK and XBP-1 splicing. In vivo effects of 4-PBA were studied in lepton-deficient $(o b / o b)$ mice, as a model of severe obesity and insulin resistance. ${ }^{56,57}$ Oral administration of 4-PBA exhibited a potent antidiabetic activity and suppressed PERK, IRE1, and JNK activation in liver and adipose tissue. 4-Phenylbutyric acid improved systemic insulin resistance and reduced the obesityinduced lipid accumulation in liver. ${ }^{58}$ Cystic fibrosis (CF), a recessive disorder, is caused by mutations of the $\mathrm{CF}$ transmembrane conductance regulator $(C F T R)$ gene disrupting its folding and trafficking and impairing chloride conductance. ${ }^{59,60}$ 4-Phenylbutyric acid in CF led to an induction of correct trafficking of a portion of mutant CFTR to the cell surface in vitro and in vivo ${ }^{32,54}$ and has been approved in humans with urea cycle deficiencies. $^{61}$

Owing to such promising results, we used the compound in a model of isolated rat pancreatic acini during supraphysiological stimulation by CCK. 4-Phenylbutyric acid preincubation reduced the activation of several ER signal pathways. We demonstrate a highly significant reduction in phospho-PERK, XBP-1 spicing downstream of IRE 1 activation, and BIP induction, a chaperone very sensitive to ER stress. Several proapoptotic pathways emitting from the ER, including CHOP expression, caspase activation, and JNK phosphorylation, were significantly down-regulated after 4-PBA pretreatment in CCKstimulated pancreatic acinar cells. Likewise, annexin V and PI staining as true early events of apoptosis were reduced. In our experiments, 4-PBA preincubation was able to influence significantly the hallmarks of CCK overstimulation. Amylase secretion significantly increased after 4-PBA treatment. Increases in enzyme secretion caused by the fatty acid 4-PBA may be explained by an optimization of ER function, folding capacity, or an increase of the efflux clearance across the apical cell membrane by induction of protein trafficking. Further, 4-PBA changes intracellular calcium signaling a main prerequisite of stimulus secretion coupling in the acini. In our experiments, 4PBA blocked the pathophysiological plateau-shaped calcium signal, whereas CCK alone stimulates the calcium released from apical granular calcium stores. To examine this more closely, we used thapsigargin, a SERCA inhibitor. Thapsigargin treatment leads to an immediate increase in intracellular calcium in acinar cells. After pretreating the cells with 4-PBA, thapsigargin's increase in intracellular calcium was significantly reduced or even abolished. To our knowledge, this is the first time this effect is described. Whether 4-PBA protects the ER calcium ATPases itself or it stabilizes the calcium channels in ER membranes is not known and was not intended to be studied and is beyond the scope of this work. However, together with the exact details of the subsequent secretion mechanisms, such as forming of secretory and zymogen granules, their transport to the apical cell pole and finally exocytosis within the context of 4-PBA treatment has to be investigated in further studies. Interestingly, 4-PBA reduced trypsin activation after CCK to approximately $50 \%$.

Overall, the protective effects of 4-PBA in pancreatic acinar cells are concordant with previous findings in other tissues, animals, and transgenic animals using the small fatty acid as chemical chaperone. 4-Phenylbutyric acid in adipose tissue reduced the expression of $\mathrm{BiP}$, phosphorylation of eukaryotic translation initiation factor $2 \alpha$ downstream of PERK phosphorylation, splicing of XBP-1, and expression of CHOP. ${ }^{37}$ It reduced the ER stress that leads to adipogenesis with a high-fat diet in animals and therefore is described as an antiobesity drug. Furthermore, 4-PBA reduced ER stress in animals with type 2 diabetes, where hyperglycemia induced $\mathrm{BiP}$ up-regulation and PERK phosphorylation. ${ }^{56}$ It further reduced ER stressassociated eukaryotic translation initiation factor $2 \alpha$ phosphorylation, caspase 12 activation, and CHOP induction caused by ischemia and reperfusion in mouse livers. ${ }^{62}$ Furthermore, it has protective effects in models of cerebral ischemic injury. ${ }^{63}$

In summary, our data show that the ER stress response of exocrine pancreatic acini to CCK overstimulation can be pharmacologically modulated. Calcium signaling, amylase secretion, and trypsin activation is influenced positively. Several apoptotic pathways are inhibited that can lead to cell damage and loss of acinar cell function. The changes induced in the present study by 4-PBA are more significant, relevant, and profound compared to the changes induced by another chemical chaperone, the bile acid tauroursodeoxycholic acid that we used in previous study. ${ }^{64} \mathrm{We}$ shed more light on the early molecular mechanisms involved in CCK overstimulation, a common model of acute experimental pancreatitis. In connection with future in vivo studies, the data may open new perspectives on the use of the orally available chemical chaperone 4-PBA as a therapeutic approach optimizing exocrine pancreas function and reducing pancreatic cell damage under conditions of overstimulation and inflammation and to keep the exocrine pancreas function after AP by reducing apoptosis and necrosis.

Thus, this treatment strategy may become important, as recent publications showed a connection between ER stress and hereditary pancreatitis as well as ER stress and excessive alcohol consumption, smoking, and metabolic disorders. ${ }^{65-69}$ All those new insights on different acute and chronic damages to the 
pancreas seem to have ER stress as a central element. Therefore, it becomes essential to treat ER stress and to ameliorate the pathophysiological part of the UPR, including induction of apoptosis and autophagy in the pancreas.

\section{ACKNOWLEDGMENT}

The authors thank U Naumann and S Rackow for their expert technical assistance.

\section{REFERENCES}

1. Case RM. Synthesis, intracellular transport and discharge of exportable proteins in the pancreatic acinar cell and other cells Biol Rev Camb Philos Soc. 1978;53:211-354.

2. Gething MJ, Sambrook J. Transport and assembly processes in the endoplasmic reticulum. Semin Cell Biol. 1990;1:65-72.

3. Berridge MJ. The endoplasmic reticulum: a multifunctional signaling organelle. Cell Calcium. 2002;32:235-249.

4. Schroder M, Kaufman RJ. Divergent roles of Irelalpha and PERK in the unfolded protein response. Curr Mol Med. 2006;6:5-36.

5. Hammond C, Helenius A. Quality control in the secretory pathway. Curr Opin Cell Biol. 1995;7:523-529.

6. Schroder M, Kaufman RJ. The mammalian unfolded protein response. Annu Rev Biochem. 2005;74:739-789.

7. Dorner AJ, Wasley LC, Kaufman RJ. Increased synthesis of secreted proteins induces expression of glucose-regulated proteins in butyrate-treated Chinese hamster ovary cells. J Biol Chem. 1989;264:20602-20607.

8. Trombetta ES, Parodi AJ. Quality control and protein folding in the secretory pathway. Annu Rev Cell Dev Biol. 2003;19:649-676.

9. Harding HP, Zhang Y, Ron D. Protein translation and folding are coupled by an endoplasmic-reticulum-resident kinase. Nature. 1999:397:271-274.

10. Travers KJ, Patil CK, Wodicka L, et al. Functional and genomic analyses reveal an essential coordination between the unfolded protein response and ER-associated degradation. Cell. 2000;101:249-258.

11. Lee AH, Iwakoshi NN, Glimcher LH. Xbp-1 regulates a subset of endoplasmic reticulum resident chaperone genes in the unfolded protein response. Mol Cell Biol. 2003;23:7448-7459.

12. Wang XZ, Harding HP, Zhang Y, et al. Cloning of mammalian Ire 1 reveals diversity in the ER stress responses. EMBO J. 1998; 17:5708-5717.

13. Nagai H, Noguchi T, Takeda K, et al. Pathophysiological roles of ask1-map kinase signaling pathways. J Biochem Mol Biol. 2007;40:1-6.

14. Oyadomari S, Mori M. Roles of chop/gadd153 in endoplasmic reticulum stress. Cell Death Differ. 2004;11:381-389.

15. Ron D, Habener JF. Chop, a novel developmentally regulated nuclear protein that dimerizes with transcription factors C/EBP and LAP and functions as a dominant-negative inhibitor of gene transcription. Genes Dev. 1992;6:439-453.

16. Morishima N, Nakanishi K, Takenouchi H, et al. An endoplasmic reticulum stress-specific caspase cascade in apoptosis. Cytochrome $c$-independent activation of caspase-9 by caspase-12. J Biol Chem. 2002;277:34287-34294.

17. Schroder M. Endoplasmic reticulum stress responses. Cell Mol Life Sci. 2008;65:862-894.

18. Urano F, Wang X, Bertolotti A, et al. Coupling of stress in the ER to activation of JNK protein kinases by transmembrane protein kinase Ire1. Science. 2000;287:664-666.

19. Williams JA, Sans MD, Tashiro M, et al. Cholecystokinin activates a variety of intracellular signal transduction mechanisms in rodent pancreatic acinar cells. Pharmacol Toxicol. 2002;91:297-303.

20. Cagliero E, Apruzzese W, Perlmutter GS, et al. Musculoskeletal disorders of the hand and shoulder in patients with diabetes mellitus. Am J Med. 2002;112:487-490.

21. Steer M. Experimental Acute Pancreatitis: Studies of Early Events That Lead to Cell Injury. 2nd ed. New York: Raven Press; 1993.
22. Kruger B, Albrecht E, Lerch MM. The role of intracellular calcium signaling in premature protease activation and the onset of pancreatitis. Am J Pathol. 2000;157:43-50.

23. van Acker GJ, Perides G, Steer ML. Co-localization hypothesis: a mechanism for the intrapancreatic activation of digestive enzymes during the early phases of acute pancreatitis. World J Gastroenterol. 2006;12:1985-1990.

24. Bhatia M, Neoptolemos JP, Slavin J. Inflammatory mediators as therapeutic targets in acute pancreatitis. Curr Opin Investig Drugs. 2001;2:496-501

25. Aho HJ, Nevalainen TJ. Experimental pancreatitis in the rat. Ultrastructure of sodium taurocholate-induced pancreatic lesions. Scand J Gastroenterol. 1980;15:417-424.

26. Hegewald G, Nikulin A, Gmaz-Nikulin E, et al. Ultrastructural changes of the human pancreas in acute shock. Pathol Res Pract. 1985;179:610-615.

27. Lerch MM, Saluja AK, Dawra R, et al. Acute necrotizing pancreatitis in the opossum: earliest morphological changes involve acinar cells. Gastroenterology. 1992;103:205-213.

28. Mizunuma T, Kawamura S, Kishino Y. Effects of injecting excess arginine on rat pancreas. $J$ Nutr. 1984;114:467-471.

29. Ji B, Chen XQ, Misek DE, et al. Pancreatic gene expression during the initiation of acute pancreatitis: identification of EGR-1 as a key regulator. Physiol Genomics. 2003;14:59-72.

30. Kubisch $\mathrm{CH}$, Sans MD, Arumugam T, et al. Early activation of endoplasmic reticulum stress is associated with arginine-induced acute pancreatitis. Am J Physiol Gastrointest Liver Physiol. 2006;291:G238-G245.

31. Lee B, Rhead W, Diaz GA, et al. Phase 2 comparison of a novel ammonia scavenging agent with sodium phenylbutyrate in patients with urea cycle disorders: safety, pharmacokinetics and ammonia control. Mol Genet Metab. 2010;100:221-228.

32. Rubenstein RC, Zeitlin PL. A pilot clinical trial of oral sodium 4-phenylbutyrate (buphenyl) in deltaf508-homozygous cystic fibrosis patients: partial restoration of nasal epithelial CFTR function. Am J Respir Crit Care Med. 1998;157:484-490.

33. Zeitlin PL, Diener-West M, Rubenstein RC, et al. Evidence of CFTR function in cystic fibrosis after systemic administration of 4-phenylbutyrate. Mol Ther. 2002;6:119-126.

34. Kubisch CH, Logsdon CD. Secretagogues differentially activate endoplasmic reticulum stress responses in pancreatic acinar cells. Am J Physiol Gastrointest Liver Physiol. 2007;292:G1804-G1812.

35. Kubisch C, Dimagno MJ, Tietz AB, et al. Overexpression of heat shock protein HSP27 protects against cerulein-induced pancreatitis. Gastroenterology. 2004;127:275-286.

36. Mooren F, Hlouschek V, Finkes T, et al. Early changes in pancreatic acinar cell calcium signaling after pancreatic duct obstruction. J Biol Chem. 2003;278:9361-9369.

37. Basseri S, Lhotak S, Sharma AM, et al. The chemical chaperone 4-phenylbutyrate inhibits adipogenesis by modulating the unfolded protein response. J Lipid Res. 2009;50:2486-2501.

38. Roque T, Boncoeur E, Saint-Criq V, et al. Proinflammatory effect of sodium 4-phenylbutyrate in deltaf508-cystic fibrosis transmembrane conductance regulator lung epithelial cells: involvement of extracellular signal-regulated protein kinase $1 / 2$ and c-Jun-NH2-terminal kinase signaling. J Pharmacol Exp Ther. 2008;326:949-956.

39. Iordache C, Duszyk M. Sodium 4-phenylbutyrate upregulates ENAC and sodium absorption in t84 cells. Exp Cell Res. 2007;313:305-311.

40. Loffing J, Moyer BD, Reynolds D, et al. PBA increases CFTR expression but at high doses inhibits $\mathrm{Cl}(-)$ secretion in Calu-3 airway epithelial cells Am J Physiol. 1999;277:L700-L708.

41. Galas MC, Lignon MF, Rodriguez M, et al. Structure-activity relationship studies on cholecystokinin: analogues with partial agonis activity. Am J Physiol. 1988;254:G176-G182.

42. Williams JA. Receptor-mediated signal transduction pathways and the regulation of pancreatic acinar cell function. Curr Opin Gastroenterol. 2008;24:573-579 
43. Treiman M, Caspersen C, Christensen SB. A tool coming of age: thapsigargin as an inhibitor of sarco-endoplasmic reticulum Ca(2+)-ATPases. Trends Pharmacol Sci. 1998;19:131-135.

44. Wu J, Kaufman RJ. From acute ER stress to physiological roles of the unfolded protein response. Cell Death Differ. 2006;13:374-384.

45. Levine B, Kroemer G. Autophagy in the pathogenesis of disease. Cell. 2008;132:27-42.

46. Tabas I, Ron D. Integrating the mechanisms of apoptosis induced by endoplasmic reticulum stress. Nat Cell Biol. 2011;13:184-190.

47. Forloni G, Terreni L, Bertani I, et al. Protein misfolding in Alzheimer's and Parkinson's disease: genetics and molecular mechanisms. Neurobiol Aging. 2002;23:957-976.

48. Collins AF, Pearson HA, Giardina P, et al. Oral sodium phenylbutyrate therapy in homozygous beta thalassemia: a clinical trial. Blood. 1995;85:43-49.

49. He B. Viruses, endoplasmic reticulum stress, and interferon responses. Cell Death Differ. 2006;13:393-403.

50. Seimon TA, Kim MJ, Blumenthal A, et al. Induction of ER stress in macrophages of tuberculosis granulomas. PLoS One. 2010;15:e12772.

51. Kaufman RJ, Back SH, Song B, et al. The unfolded protein response is required to maintain the integrity of the endoplasmic reticulum, prevent oxidative stress and preserve differentiation in beta-cells. Diabetes Obes Metab. 2010;12(suppl 2):99-107.

52. Cohen FE, Kelly JW. Therapeutic approaches to protein-misfolding diseases. Nature. 2003;426:905-909.

53. Perlmutter DH. Chemical chaperones: a pharmacological strategy for disorders of protein folding and trafficking. Pediatr Res. 2002;52:832-836.

54. Rubenstein RC, Egan ME, Zeitlin PL. In vitro pharmacologic restoration of CFTR-mediated chloride transport with sodium 4-phenylbutyrate in cystic fibrosis epithelial cells containing delta f508-CFTR. J Clin Invest. 1997;100:2457-2465.

55. Engin F, Hotamisligil GS. Restoring endoplasmic reticulum function by chemical chaperones: an emerging therapeutic approach for metabolic diseases. Diabetes Obes Metab. 2010;12(suppl 2):108-115.

56. Ozcan U, Yilmaz E, Ozcan L, et al. Chemical chaperones reduce ER stress and restore glucose homeostasis in a mouse model of type 2 diabetes. Science. 2006;313:1137-1140.
57. Ozcan U, Cao Q, Yilmaz E, et al. Endoplasmic reticulum stress links obesity, insulin action, and type 2 diabetes. Science. 2004;306:457-461.

58. Gregor MF, Hotamisligil GS. Thematic review series: adipocyte biology. Adipocyte stress: the endoplasmic reticulum and metabolic disease. $J$ Lipid Res. 2007;48:1905-1914.

59. Gabriel SE, Clarke LL, Boucher RC, et al. Cftr and outward rectifying chloride channels are distinct proteins with a regulatory relationship. Nature. 1993;363:263-268.

60. Stutts MJ, Canessa CM, Olsen JC, et al. CFTR as a cAMP-dependent regulator of sodium channels. Science. 1995;269:847-850.

61. Newmark HL, Young CW. Butyrate and phenylacetate as differentiating agents: practical problems and opportunities. J Cell Biochem. 1995;22:247-253.

62. Vilatoba M, Eckstein C, Bilbao G, et al. Sodium 4-phenylbutyrate protects against liver ischemia reperfusion injury by inhibition of endoplasmic reticulum-stress mediated apoptosis. Surgery. 2005;138:342-351.

63. Qi X, Hosoi T, Okuma Y, et al. Sodium 4-phenylbutyrate protects against cerebral ischemic injury. Mol Pharmacol. 2004;66:899-908.

64. Malo A, Kruger B, Seyhun E, et al. Tauroursodeoxycholic acid reduces endoplasmic reticulum stress, trypsin activation, and acinar cell apoptosis while increasing secretion in rat pancreatic acini. Am J Physiol Gastrointest Liver Physiol. 2010;299:G877-G886.

65. Kereszturi E, Szmola R, Kukor Z, et al. Hereditary pancreatitis caused by mutation-induced misfolding of human cationic trypsinogen: a novel disease mechanism. Hum Mutat. 2009;30:575-582.

66. Lugea A, Waldron RT, French SW, et al. Drinking and driving pancreatitis: links between endoplasmic reticulum stress and autophagy. Autophagy. 2011;7:783-785.

67. Pandol SJ, Gorelick FS, Gerloff A, et al. Alcohol abuse, endoplasmic reticulum stress and pancreatitis. Dig Dis. 2011;28:776-782.

68. Pandol SJ, Gorelick FS, Lugea A. Environmental and genetic stressors and the unfolded protein response in exocrine pancreatic function-a hypothesis. Front Physiol. 2011;2:8.

69. Alexandre M, Pandol SJ, Gorelick FS, et al. The emerging role of smoking in the development of pancreatitis. Pancreatology. 2011;11:469-474 